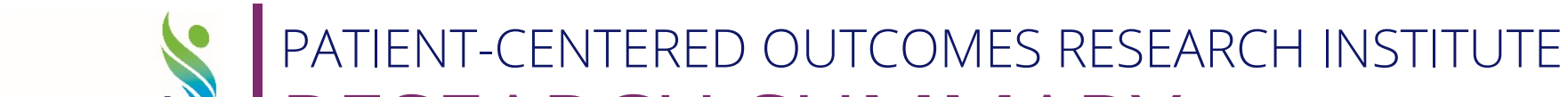 pcori). RESEARCH SUMMARY
}

\section{Comparing Treatment for Ductal Carcinoma In Situ (DCIS) with or without Sentinel Lymph Node Biopsy}

Principal investigator

Shiyi Wang, MD, PhD
Organization

Yale University

\section{What was the research about?}

Ductal carcinoma in situ, or DCIS, makes up about 25 percent of new breast cancer diagnoses. In DCIS, the cancer cells occur inside milk ducts. DCIS may or may not turn into invasive cancer, which spreads and causes illness.

Women with DCIS often have breast-conserving surgery to treat DCIS. This surgery removes the abnormal cells and some of the tissue around them. Sometimes the surgeon also removes some lymph nodes around the tumor, called sentinel lymph nodes, to test if the cancer has spread. This sentinel lymph node biopsy may cause side effects like pain or infection.

In this study, the research team compared older women with DCIS who'd had surgery with and without sentinel lymph node biopsy. They wanted to learn if the biopsy

- Increased side effects

- Reduced the spread of cancer

- Helped patients live longer

The research team looked at four types of side effects in the short term and in the longer term. The side effects included swelling, infections, pain, and fluid under patients' skin where they'd had surgery.

\section{What were the results?}

Short-term side effects. Women who had sentinel lymph node biopsy were more likely to have one or more of the four types of side effects within nine months of DCIS diagnosis compared with women who didn't have a biopsy.

Long-term side effects. Compared with women who didn't have a biopsy, those who had sentinel lymph node biopsy were also more likely to have swelling up to 12 years after DCIS diagnosis. The two groups didn't differ in how likely they were to have infections, pain, and fluid under the skin.

Health effects. The study didn't show differences between the two groups of women in how often the cancer spread or how long they lived after DCIS diagnosis.

\section{Who was in the study?}

The research team used data from a national Medicare database that included 7,127 women with DCIS who were between the ages of 67 and 94 and who had breast-conserving surgery. Of these women, 88 percent were white, 8 percent were black, and 5 percent were other races; also, 5 percent were Hispanic. The average age was 75.

\section{What did the research team do?}

The research team compared the Medicare data for women diagnosed with DCIS who did and didn't have sentinel lymph node biopsy. They looked at side 
effects that happened within 9 months after diagnosis, and at side effects, cancer spread, and length of life up to 12 years after diagnosis. The team took into account other things that might affect how likely patients were to have side effects, such as their age, year of diagnosis, and tumor size.

An advisory group of breast cancer survivors and doctors who treat cancer helped design the study.

\section{What were the limits of the study?}

Most women in the study were white and older than 66. Results may differ if the research team included women from different backgrounds. The women who had biopsies may have been different from the women who didn't have them. The data didn't include some information about patients, like other health problems, which could have affected the results.

Future research could include women who are younger and from more diverse backgrounds. Research could also include information about other health problems that might affect the results.

\section{How can people use the results?}

Older women and doctors can consider the results when making treatment decisions for DCIS.

To learn more about this project, visit www.pcori.org/Wang214. 